\title{
Morris' Pariser Programm einer wissenschaftlichen Philosophie
}

Thomas Mormann

\begin{abstract}
Einer der institutionellen Höhepunkte in der Begegnung von österreichischer "wissenschaftlicher Philosophie" und französischer "Philosophie scientifique" in der ersten Hälfte des 20. Jahrhunderts war der "Erste Internationale Kongress für Einheit der Wissenschaft", der im September 1935 in Paris stattfand. In meinem Beitrag geht es um eine Episode dieses philosophischen Großereignisses, deren Protagonist der amerikanische Philosoph und Semiotiker Charles William Morris war. Auf dem Kongreß präsentierte sein Programm einer umfassenden praxisorientierten wissenschaftlichen Philosophie, das er 1937 in Logical Positivism, Pragmatism and Scientific Empiricism ausarbeitete. Morris zielte auf eine Synthese von Formalismus, Pragmatismus und traditionellem Empirismus, welche die Vorteile dieser Ansätze bündeln und ihre Einseitigkeiten und Unzulänglichkeiten vermeiden sollte. Der Kern seines Ansatzes war eine umfassende Theorie des Bedeutungsbegriffes. Vom Begriff der Bedeutung aus wollte er die vorhandenen Differenzen und die Möglichkeiten eines zukünftigen Zusammengehens von logischem Empirismus und Pragmatismus analysieren. Gegen das zu enge logisch-empiristische Verständnis von Philosophie als Syntax der Wissenschaftssprache plädierte Morris für einen "wissenschaftlichen Pragmatismus", der vier Ebenen umfaßte, nämlich (1) Philosophie als Wissenschaftslogik (Carnap), (2) Philosophie als Bedeutungsklärung (Peirce), (3) Philosophie als empirische Wertlehre (Dewey), und Philosophie als empirische Kosmologie (Whitehead).
\end{abstract}

Abstract. L'une des expressions institutionnelles les plus importantes à la rencontre de la "wissenschaftlicher Philosophie" autrichienne et la "Philosophie scientifique", dans la première moitié du XXe siècle, a été le "Premier Congrès International pour l'Unité de la Science" qu'il a eu lieu à Paris en septembre 1935. Dans ma contribution j'analyse un épisode de cet événement, dont le protagoniste a été le semiotique et philosophe américain Charles William Morris. Dans le Congrès il a présenté son programme pour une philosophie scientifique comprehensive orientée à la pratique, qu'il a développée ensuite en 1937 dans son Logical 
Positivism, Pragmatism and Scientific Empiricism. Morris a construit une synthèse de formalisme, de pragmatisme et d'empirisme traditionnel, qui devait rendre cohérent les avantages de ces points de vue et éviter ses insuffisances. Le noyau de son point de vue était une théorie générale du concept de signification. À partir du concept de signification il essayait d'analyser les différences existantes et les possibilités pour une unité future entre l'empirisme logique et le pragmatisme. En face d'une interprétation empiriste logique étroite de la philosophie comme syntaxe du langage de la science, Morris plaidait pour un "pragmatisme scientifique" qu'il concrétisait dans quatre niveaux, à savoir : (1) philosophie comme logique de la science (Carnap), (2) philosophie comme élucidation de la signification (Peirce), (3) philosophie comme théorie empirique des valeurs (Dewey), et (4) philosophie comme cosmologie empirique (Whitehead).

Abstract: One of the institutional highlights of the encounter between Austrian "wissenschaftliche Philosophie" and French "philosophie scientifique" in the first half of the $20^{\text {th }}$ century was the "First International Congress for Unity of Science" that took place 1935 in Paris. In my contribution I deal with an episode of the philosophical mega-event whose protagonist was the American philosopher and semiotician Charles William Morris.

At the Paris congress he presented his programme of a comprehensive, practice-oriented scientific philosophy and, in a more elaborated version he published it two years later in Logical Positivism, Pragmatism and Scientific Empiricism (Morris 1937). Morris aimed at a synthesis of formalism, pragmatism, and traditional empiricism that combined the virtues of these accounts while avoided their shortocmings. The core of approach was a comprehensive theory of the concept of meaning. Through an analysis of the concept of meaning he sought to sort out the existing differences and the options for a possible future rapprochment between logical empiricism and pragmatism. Against the overly narrow logical empiricist understanding of philosophy as the syntax of the language of science Morris argued for a "scientific pragmatism" that comprised four levels: (1) Philosophy as Logic of Science, (2) Philosophy as Clarification of Meaning (Peirce), (3) Philosophy as Empirical Axiology (Dewey), and (4) Philosophy as Empirical Cosmology (Whitehead).

1. Einleitung. Einer der institutionellen Höhepunkte in der Begegnung von österreichischer "wissenschaftlicher Philosophie" und französischer "Philosophie scientifique" in der ersten 
Hälfte des 20. Jahrhunderts war der "Erste Internationale Kongress für Einheit der Wissenschaft" - "Congrès International de Philosophie Scientifique", der vor etwas mehr als 75 Jahren im September 1935 in Paris stattfand.

In dieser Arbeit möchte ich auf eine Episode dieses philosophischen Großereignisses eingehen, die bisher nur geringe Aufmerksamkeit in der Philosophiegeschichte gefunden hat. Ihr Protagonist war der amerikanische Philosoph und Semiotiker Charles William Morris. Genauer gesagt, geht es um ein Projekt, das man als “Morris' Pariser Programm einer wissenschaftlichen Philosophie” bezeichnen könnte. Ähnlich wie Neuraths Wortschöpfung "Wiener Kreis" etwas Positives, Interessantes und Attraktives suggerieren sollte, möchte ich mit der Bezeichnung "Pariser Programm" positive Assoziationen wecken. Meine These ist also, daß dieses Programm verdient, nicht in völlig in Vergessenheit zu geraten, auch wenn es mit der zeitgenössischen Wissenschaftsphilosophie nicht ohne weiteres kompatibel sein mag. Ich halte Morris' Pariser Programm für einen Ansatz der Wissenschaftsphilosophie, der nicht nur philosophiehistorisches Interesse beanspruchen darf, sondern der es darüber hinaus auch verdiente, in den aktuellen Diskussionen über die Rolle der Philosophie in unserer von den Wissenschaften geprägten Kultur zur Kenntnis genommen zu werden.

Morris hat sein Programm bis an sein Lebensende vertreten, auch wenn zuletzt sowohl die Logischen Empiristen wie auch die anderen amerikanischen Pragmatisten seinem Projekt nur noch wenig Interesse entgegenbrachten.

Das "offizielle" Programm des Pariser Kongresses war natürlich nicht das von Morris, sondern Neuraths Programm der "Einheit der Wissenschaft". In einem allgemeinen Sinne paßte Morris' Programm, das auf eine "Vereinigung" von Wiener Logischem Empirismus und amerikanischem Pragmatismus zielte, recht gut in diesen Rahmen. Schaut man genauer hin, war das Verhältnis von Morris' Programm zum Kern des einheitswissenschaftlichen Programms, eben zu Neuraths Enzyklopädismus, jedoch keineswegs einfach und unproblematisch.

Die ausführlichste Ausarbeitung von Morris' Programm einer umfassenden praxisorientierten wissenschaftlichen Philosophie, findet sich in dem schmalen Buch Logical Positivism, Pragmatism and Scientific Empiricism (Morris 1937, im Folgenden LPS). ${ }^{1}$ Der letzte Teil dieser Broschüre, Semiotic and Scientific Empiricism, ist identisch mit Morris' Beitrag zum Kongress

\footnotetext{
${ }^{1}$ Morris' Broschüre ist anscheinend die einzige Monographie der damals bei Hermann erschienenen Actualites scientifiques et industrielles, die in Englisch (und nicht in Französisch) veröffentlicht worden ist. Es wäre interessant herauszufinden, über welche Verbindungen mit Vertretern der "philosophie scientifique" Morris verfügte, die es inm ermöglichten, sein Buch bei Hermann auf Englisch herauszubringen.
} 
von 1935. Er umfaßt etwas mehr als ein Drittel des Gesamttextes. Auf den letzten Zeilen dieses Textes faßt Morris die allgemeine Intention seines wissenschaftsphilosophischen Programms so zusammen:

The program thus sketched ... envisages the expansion of the current emphasis upon logical analysis into a general interest in meaning-analysis, and this in turn into semiotic. It sees the current positivisms rounding themselves out into a scientific empiricism which by doing justice to the three dimensions of meaning is able to unite the attitudes of formalism, pragmatism, and traditional empiricism, and at the same time to give promise of resolving the inadequacies which have beset previous forms of empiricism. (Morris $(1936,56)$ und $(1937,71)$ ).

Noch 1939, auf dem letzten großen Kongress für "Einheit der Wissenschaft" der in Cambridge/Massachusetts an der Harvard University abgehalten wurde, engagierte sich Morris für dieses Programm. Darauf soll später noch genauer eingegangen werden. Zum letzten Mal versuchte er in seinem Beitrag zum Carnap gewidmeten "Schilpp-Band", Carnap für sein seit den 1930er Jahren propagiertes Vereinigungs- oder Annäherungsprogramm zwischen Pragmatismus und Logischem Empirismus zu gewinnen, ohne jedoch in Carnaps Replik mehr als lauwarme Zustimmung zu ernten (cf. Morris 1963, Carnap 1963).

Mein Beitrag ist folgendermaßen gegliedert: Im nächsten Abschnitt sollenn zunächst die wesentlichen Punkte des Pariser Programms, wie es Morris in LPS formulierte, vorgestellt werden. Dann möchte ich, um den Pariser Kontext von Morris' Programm in seinen Grundzügen zu umreißen, eingehen auf zwei konkurrierende Programme, die ebenfalls auf dem Kongress präsentiert wurden, nämlich Carnaps Wissenschaftslogik und Neuraths Enzyklopädismus. Die Auseinandersetzung mit beiden bestimmte Morris' philosophische Arbeit in den folgenden Jahrzehnten in wesentlichen Aspekten. Aus Morris' pragmatischer Perspektive erscheinen sie als Verkürzungen seines Ansatzes. Morris' eigentümliches Verständnis der komplementären Beziehung von Pragmatismus und logischem Empirismus manifestierte sich in seinem Begriff des "variablen Apriori", das er als eine komplexe Beziehung zwischen Regeln, Zwecken und Daten konzipierte. Bei seinen logisch-empiristischen Diskussionspartnern auf dem Kongress und darüber hinaus scheint dieser Begriff jedoch auf wenig Gegenliebe gestoßen zu sein. 
Die in Paris begonnenen Diskussionen zwischen europäischen logischen Empiristen und amerikanischen Pragmatisten setzten sich in den folgenden Jahren in den USA fort, ohne ein konklusives Ergebnis zu zeitigen. Einige Stationen dieser Entwicklung sollen genauer betrachtet werden. Abschließend möchte ich versuchen, zu einer zumindest vorläufigen Gesamtbeurteilung von Morris' Programm im Kontext der wissenschaftsorientierten Philosophie des 20. Jahrhunderts zu kommen.

2. Morris' logisch-empiristischer Pragmatismus. Für Morris war der Pariser Kongress Ausdruck einer neuen Phase der Zusammenarbeit von Philosophie und Wissenschaft. In dieser neuen Phase, so Morris, trete ein neuer Typ des Gelehrten auf den Plan, den er als "logischen Wissenschaftler" oder "wissenschaftlichen Logiker" (logician-scientist) charakterisierte: Dieser Gelehrte neuen Typs gebe sich weder mit dem leeren Gerede der traditionellen philosophischen Spekulationen zufrieden, noch finde er sich ab mit der in den Wissenschaften verbreiteten geistlosen Wiederholung logisch nicht analysierter Begriffe und Sätze.

Mit dem Programm der Einheitswissenschaft kehre, so Morris, das alte Ideal einer Philosophie und empirische Wissenschaften umfassenden Gesamtwissenschaft in neuer Form zurück. Wissenschaftliche Philosophie und philosophische Wissenschaft liefen damit auf dasselbe hinaus. ${ }^{2}$ Beide hättem letztlich dasselbe Ziel, nämlich den Aufbau einer rationalen, aufgeklärten, und durch die Wissenschaften geprägten Kultur und Gesellschaft (LPS, 3).

LPS ist eine Kompilation von Arbeiten, die Morris um die Mitte der dreißiger Jahre als Zeitschriftenaufsätze und Kongressbeiträge veröffentlicht hatte. LPS besteht aus fünf Kapiteln:

I. Philosophy of Science and Science of Philosophy (Philosophy of Science 2, 1935)

II. The Concept of Meaning in Pragmatism and Logical Positivism (Proceedings of the $8^{\text {th }}$ International Congress of Philosophy in Prague, 1934)

III. Pragmatism and Metaphysics (The Philosophical Review XLIII, 1934)

IV. The Relation of the Formal and Empirical Sciences within Scientific Empiricism (Erkenntnis 5, 1935)

V. Semiotic and Scientific Empiricism (Beitrag zum Pariser Kongress 1935)

${ }^{2}$ Morris bezieht sich mit dieser These ausdrücklich auf das emphatische Diktum Schlicks in "Die Wende der Philosophie". 
Für Morris war Philosophie nur möglich in Beziehung auf die Wissenschaften. Für sich allein degenerierte Philosophie zur haltlosen metaphysischen Spekulation. Sie besaß keine Methoden, die nur ihr und nicht den Wissenschaften zur Verfügung standen. Ein synthetisches, auf Anschauung begründetes a priori lehnte er deshalb strikt ab, wohl aber entwickelte er in The Relation of the Formal and the Empirical Sciences within Scientific Empiricism (LPS, IV) eine interessante Theorie eines variablen analytischen a priori, worauf in Abschnitt 4 noch genauer eingegangen werden soll.

Der Kern einer wissenschaftlichen Philosophie war für Morris eine umfassende Theorie des Bedeutungsbegriffes. Dieser Begriff war die Basis, von der aus er die vorhandenen Differenzen und die Möglichkeiten eines zukünftigen Zusammengehens von Empirismus und Pragmatismus analysieren wollte. (LPS, 23) Ähnlich wie andere amerikanische Pragmatisten warf Morris den logischen Empiristen einen verkürzten Begriff von Bedeutung vor, der wissenschaftliche Philosophie zu eng als syntaktische Theorie der Wissenschaftssprache auffaßte.

Aus Peirce' dreistelligem Zeichenbegriff (cf. Apel 1974), wonach jedes Zeichen drei auf einander bezogene, aber nicht aufeinander reduzierbare Aspekte besitzt, ergaben sich für Morris als Teiltheorien einer allgemeinen semiotisch orientierten Philosophie Syntax, Semantik, und Pragmatik, die jeweils einen Zeichenaspekt in den Vordergrund stellen: Die Syntax befaßt sich mit den Beziehungen von Zeichen unter einander, die Semantik thematisiert die Beziehungen zwischen Zeichen und Gegenständen, und in der Pragmatik geht es eine Theorie der praktischen Zeichenverwendung. Aus einer etwas anderen Perspektive ergaben sich daraus für ihn die philosophischen Ansätze des Formalismus, Empirismus und des Pragmatismus. Gegen das seiner Meinung nach zu enge Verständnis von Philosophie als Syntax der Wissenschaftssprache plädierte Morris für einen "wissenschaftlichen Pragmatismus", der vier verschiedene Ebenen umfaßte, die er grob durch die Namen Carnap, Peirce, Dewey, and Whitehead kennzeichnete (LPS, 8ff.):

1. Philosophie als Wissenschaftslogik (Carnap)

2. Philosophie als Bedeutungsklärung (Peirce)

3. Philosophie als empirische Wertlehre (Dewey)

4. Philosophie als empirische Kosmologie (Whitehead)

In diesem Schema figurierte Carnaps Wissenschaftslogik als unterste Ebene einer umfassenden, nicht nur syntaktische Aspeke der Wissenschaftssprache thematisierenden wissen- 
schaftlichen Philosophie. Im Vergleich zu den höheren Ebenen war die syntaktische Ebene durch den höchsten Grad an Gewißheit und Präzision ausgezeichnet. Morris gab ohne Umschweife zu, daß der Übergang von "Carnap" zu "Whitehead" mit einer Verringerung von Exaktheit und Gewißheit einhergehe (LPS, 19). Er war jedoch überzeugt, eine umfassende wissenschaftliche Philosophie müsse diesen Preis zahlen, da erkenntnismäßige Gewißheit nicht das einzige Kriterium sein dürfte, Philosophie sei für alle Aspekte der Bedeutung wissenschaftlicher und nichtwissenschaftlicher Zeichensysteme zuständig.

Morris war sich also darüber im klaren, daß man sich, um ein etwas schiefes Bild zu benutzen, ab und zu "begrifflich die Hände schmutzig machen" mußte, wenn man sich mit Problemen befaßte, die die "höheren" Aspekte der Philosophie betrafen. Nicht immer seien in der Philosophie "reine" Begriffe verfügbar. Eine "Flucht auf die eisigen Firne der Logik" war also für ihn nicht die richtige Reaktion des wissenschaftlichen Philosophen. In Hinsicht auf die Unvermeidbarkeit "unreiner" Begriffe berührten sich seine Auffassungen mit denen Neuraths, der ja des öfteren vor der "pseudorationalistischen" Einstellung warnte, man könnte in der Philosophie immer und überall mit "sauberen” Begriffen arbeiten. Damit stellte sich Morris' wissenschaftlicher Empirismus zumindest implizit gegen Carnaps Vorschlag einer Arbeitsteilung, wonach sich sauber zwischen Wissenschaftslogik und anderen Gebieten einer umfassenden Wissenschaftstheorie trennen lasse, die sich mit psychologischen, soziologischen, oder historischen Aspekten der Wissenschaft befassten. In der Wissenschaft, so Morris, gebe es keinen absoluten Bruch zwischen Theorie und Praxis, deshalb gebe es auch keinen überzeugenden Grund für die Annahme, das könnte in einer "wissenschaftlichen" Philosophie anders sein. Gesellschaftlich, politisch, und allgemein praktisch relevante Aspekte der Wissenschaft aus der Wissenschaftsphilosophie im eigentlichen Sinne auszuschließen, war für Morris ein Zeichen ethischer Verantwortungslosigkeit:

It is important to distinguish dreams and fancies from expectations and proposals based on whatever data is available and controllable by new data obtained in use. It would be a signal instance of ethical irresponsibility to relinquish the demands which the need for a philosophically disciplined imagination imposes, and to turn the world over to the exclusive control of dreamers, adventurers, men of action, and technicians. (LPS, 20) 
Der sich seiner gesellschaftlichen Verantwortung bewußte Philosoph, so Morris, tue in einem weiteren Sinne genau das, was auch der Logiker tue, wenn er geeignete Schlußregeln auswähle, und was der Wissenschaftler innerhalb seines Tätigkeitsbereiches tue, wenn er nach einem begrifflichen System suche, das zu den Tatsachen am besten paßt und das die genaueste Bestimmung dessen erlaubt, was in der Zukunft zu erwarten ist:

Philosophy is the most general science and the widest vision, - and the one because it is the other (LPS, Kapitel I, 20).

Man kann Morris' Programm den Auffassungen anderer Pragmatisten wie Clarence Irvine Lewis oder Abraham Kaplan an die Seite stellen, die alle auf je verschiedene Weise gegen die logistische Austrocknung der wissenschaftlichen Philosophie durch den received view des logischen Empirismus angegangen sind. In prägnanter Form hat Lewis diesen für jede pragmatische Wissenschaftsphilosophie fundamentalen Zusammenhang von Wissen, Handlung und Wertung so formuliert:

Knowledge, action, and evaluation are essentially connected. The primary and pervasive significance of knowledge lies in its guidance of action; knowing is for the sake of doing. And action, obviously, is rooted in evaluation. For a being which did not assign comparative values, deliberate action would be pointless; and for one which did not know, it would be impossible. Conversely, only an active being could have knowledge, and only such a being could assign values to anything beyond his own feelings. (Lewis 1946, An Analysis of Knowledge and Valuation, 5)

Logische Empiristen carnapianischer Observanz haben sich immer geweigert, diesen Zusammenhang ohne Wenn und Aber anzuerkennen. Auch wenn bei Carnap Spuren pragmatischen Denkens zu finden sein mögen, kann man ihm keine genuin pragmatische Konzeption zuschreiben. Für Morris lief der Verzicht der Wissenschaftsphilosophie, sich mit Problemen der wissenschaftlichen Praxis und Pragmatik auseinander zu setzen, darauf hinaus, "die Welt der Kontrolle von Träumern, Abenteurern, Technikern und Männern der Tat zu überlassen" (LPS, 20) - mit den zu erwartenden Folgen. 
3. Das Pariser Programm im Kontext. Morris' Ansatz konkurrierte auf dem Kongreß mit zwei anderen Programmen einer wissenschaftlichen Philosophie, nämlich Carnaps Wissenschaftslogik, das dieser auf dem Kongress in seinem Beitrag Von der Erkenntnistheorie zur Wissenschaftslogik (Carnap 1936) vorstellte und dem Enzyklopädieprogramm, welches Neurath in dem Vortrag Einzelwissenschaften, Einheitswissenschaft, Pseudorationalismus (Neurath 1936) präsentierte.

Morris' Programm einer umfassenden pragmatischen wissenschaftlichen Philosophie stand in Gegensatz zu Carnaps Wissenschaftslogik. Gegenläufig zu Morris beschrieb Carnap in seinem Kongreßbeitrag den jüngsten Fortschritt der Wissenschaftsphilosophie als einen Destillationsprozeß, in dem Schritt für Schritt aus der metaphysisch kontaminierten Philosophie eine "reine" Wissenschaftslogik als keimfreier Kern der Philosophie destilliert worden sei:

Die bisherigen Hauptphasen der Entwicklung der wissenschaftlichen Philosophie kann man vielleicht so charakterisieren: Zuerst handelte es sich um die Überwindung der Metaphysik, um den Übergang von der spekulativen Philosophie zur Erkenntnistheorie. Der zweite Schritt bestand in der Überwindung des synthetischen Apriori; er führte zur empiristischen Erkenntnistheorie. ... Die Aufgabe unserer gegenwärtigen Arbeit scheint mir nun in dem Übergang von der Erkenntnistheorie zur Wissenschaftslogik zu bestehen. Hierbei wird die Erkenntnistheorie ... gereinigt und in ihre Bestandteile aufgelöst. (Carnap 1936, 37)

"Reinigung" führte zu einer Arbeitsteilung: Die Untersuchung der empirischen Tatsachen war Aufgabe der realwissenschaftlich empirischen Forschung, die der Sprachformen Aufgabe der logischen, d.h. der syntaktischen Analyse. Ein dritter Gegenstandsbereich neben dem empirischen und dem logischen war nicht zugelassen. Damit zerfiel die Wissenschaftsphilosophie in einen genuin philosophischen Teil, die "Wissenschaftslogik", und in gewisse andere empirische Disziplinen wie Wissenschaftsgeschichte, Wissenschaftspsychologie oder Wissenschaftssoziologie, über die sich Carnap wenig Gedanken machte.

Nach Carnaps Meinung war sein Programm eines Übergangs von der Erkenntnistheorie zur Wissenschaftslogik eigentlich gar kein Programm mehr, sondern eine Tatsache, die es nur noch explizit zu machen gilt: 
Es handelt sich nur noch darum, daß wir uns diesen Übergang, der sich schon vollzieht, bewußt machen, um ihn klar und methodisch durchzuführen. (Carnap $1935,41)$

Carnaps Einstellung zu Morris' Projekt einer Vereinigung von Logischem Empirismus und amerikanischen Pragmatismus war nie von großem Enthusiasmus geprägt. Er versuchte dem Problem, inwieweit eine Übereinstimmung zwischen Pragmatisten und Empiristen erreicht worden sei oder in Zukunft erreichbar sei, diskret auszuweichen. Eine offensichtliche, niemals überwundene Differenz zwischen beiden Ansätzen blieb der strikte Nonkognitivismus des logischen Empirismus des Wiener Kreises, d.h. die strikte Trennung faktischer und wertender Komponenten. Carnap hätte deshalb Lewis' im vorigen Abschnitt erwähntes Diktum über den Zusammenhang von Wissen, Tätigkeit und Bewertung gewiß abgelehnt. Für ihn hatten pragmatische Überlegungen über Vor- und Nachteile von Sprachen oder linguistischontologischen Rahmen ihren Platz nicht innerhalb der Wissenschaftslogik. Die Aufgabe des carnapianischen Wissenschaftsphilosophen als eines "neutralen" Sprachingenieurs bestand einzig darin, Sprachen zu konstruieren - diese als geeignet oder ungeeignet für bestimmte Zwecke anzusehen, blieb den "Praktikern" überlassen, seien diese nun Wissenschaftler, Politiker, oder "Anwender" welcher Couleur auch immer.

Auf einer noch fundamentaleren Ebene kann man die Differenzen zwischen Carnaps logischem Empirismus und dem Pragmatismus von Dewey, Lewis, und Morris auf ihre gegensätzlichen Auffassungen darüber zurückführen, was überhaupt Wissenschaft sei. Für Carnap war Wissenschaft ein System theoretischen Wissens - ein System begründbarer Aussagen (Carnap 1935, 32). Für Dewey hingegen, um ihn als radikalsten Vertreter einer genuin pragmatistischen Wissenschaftsphilosophie hervorzuheben, war Wissenschaft eine Tätigkeit, in der es darum ging, Probleme zu lösen. Daraus ergab sich, daß Bewertungen vielfältiger Art für die Erzeugung wissenschaftlichen Wissens wesentlich waren. Carnap hingegen insistierte auf einer strikten Trennung zwischen wissenschaftlichem Wissen und Werturteilen.

Außer mit Carnaps Wissenschaftslogik sah sich Morris' Programm auf dem Kongress auch mit Neuraths Enzyklopädieprojekt konfrontiert, dessen Details hier als bekannt vorausgesetzt werden sollen (vgl. Nemeth und Roudet 2005). Man kann mit einigem Recht behaupten, daß auf dem Pariser Kongress Neuraths Projekt in einem gewissen Sinne auf dem Zenit seines Ansehens stand, auch wenn es noch nicht einmal in Ansätzen verwirklicht worden war: immerhin gab "Enzyklopädie der Einheitswissenschaft" ja das Thema des Kongresses vor. 
Darüber hinaus sprach sich der Kongreß (auf Morris' Antrag hin) offiziell dafür aus, das von Neuraths Mundaneum Institute in Den Haag in Aussicht genommene Projekt einer Enzyklopädie zu unterstützen und daran mitzuarbeiten (vgl. Erkenntnis 5 (1935), 407-408). Dieser Enthusiasmus für das Enzyklopädieprojekt hielt jedoch nicht lange vor. Auf den folgenden Kongressen ließ die Begeisterung für Neuraths Unternehmung zusehends nach (vgl. Soulez 2005, 138).

Es gibt jedoch einen wenig beachteten Aspekt von Neuraths Programm, der zwar für das Programm selbst keine große Bedeutung hatte, den man aber im Zusammenhang mit Morris' erwähnen sollte. Neurath versuchte nämlich, sein Enzyklopädieprogramm mit Carnaps Programm der Wissenschaftslogik zu verbinden. Auf diese Weise hoffte er wohl, eine logischempiristische Einheitsfront gegen andere konkurrierende Strömungen, innerhalb der wissenschaftlichen Philosophie, insbesondere gegen den amerikanischen Pragmatismus bilden zu können:

An die von Reichenbach und Carnap gekennzeichneten Etappen: von der Metaphysik zur Erkenntnistheorie, von der Erkenntnistheorie zur Wissenschaftslogik, kann man als nächste Etappe den Schritt zur Einheitswissenschaft anschliessen, die mithilfe der Wissenschaftslogik aufgebaut, an die Stelle umfassender Schau, wie sie von der Metaphysik versucht wurde, die planmäßige Zusammenfassung alles dessen setzt, was wir an wissenschaftlichen Sätzen erarbeitet haben. (Neurath 1936,57 )

Die Idee eines "kombinierten Ansatzes" war nicht neu: Bereits im Manifest von 1929 hatte Neurath für Carnaps Konstitutionstheorie eine entsprechende Rolle vorgesehen. Danach sollte die Konstitutionstheorie den Rahmen abgeben für die Anwendung der logischen Analyse, der alle wissenschaftlichen Begriffe zu unterziehen waren, bevor sie ihren Platz im System der Einheitswissenschaft einnehmen konnten. Daraus wurde nie etwas, um 1930 verschwand die Konstitutionstheorie stillschweigend aus Neuraths Begriffsarsenal und wurde kurze Zeit später durch die Wissenschaftslogik, wie sie Carnap in Logische Syntax der Sprache entwickelt hatte, ersetzt. Neurath propagierte also eine Art Arbeitsteilung zwischen Wissenschaftslogik und Enzyklopädie. Auch dieses Projekt wurde nie realisiert.

Ganz unabhängig von einer eventuellen Frontstellung zu Morris' pragmatistischem Programm kann man die von Neurath propagierte Synthese von Enzyklopädismus und Wissenschafts- 
logik als Antwort auf ein Problem ansehen, das den Logischen Empirismus immer geplagt hat, nämlich das Problem des prekären Bezugs der logisch-empiristischen Wissenschaftsphilosophie zur Realität der Wissenschaft und insbesondere zur wissenschaftlichen Praxis:

Man gibt oft der Befürchtung Ausdruck, der logische Empirismus könnte in leere Scholastik und Dogmatismus verfallen. Wir entgehen solchen Gefahren ... um so leichter, je mehr man sich um die Weiterführung wissenschaftlicher Arbeit im weitesten Umfang bemüht. (Neurath 1936, 57)

Als Rezept gegen eine solche Fehlentwicklung empfahl Neurath eine naturalistische Interpretation der logischen Analyse. Die logische Analyse sollte ihre Nützlichkeit in der Anwendung und in der Zusammenarbeit mit den empirischen Wissenschaften zeigen. Ob ihr das jemals gelungen ist, läßt sich füglich bezweifeln.

Ungeachtet der Tatsache, daß Morris mit Neurath über lange Jahre loyal im Enzyklopädieprogramm zusammenarbeitete, scheint es keine inhaltliche Diskussion zwischen Neurath und Morris über die Beziehung zwischen Pariser Programm und Enzyklopädismus gegeben zu haben. Neurath blieb jedenfalls zeitlebens bei einem strikten, antipragmatischen Nonkognitivismus und zeigte nicht das geringste Interesse für semiotische Fragestellungen, die Morris am Herzen lagen. Auch Morris' Diskussion mit Carnap über das problematische Verhältnis von logischem Empirismus und Pragmatismus, die sich bis in die sechziger Jahre hinzog, verlief letztlich im Sande.

Bevor auf diesen späten Ausläufer der Diskussion zwischen Logischem Empirismus und Pragmatismus eingegangen werden soll, möchte ich zunächst eine merkwürdige Episode der Auseinandersetzung zwischen beiden Strömungen behandeln, die in der Philosophiegeschichte fast völlig unbemerkt geblieben ist, obwohl es sich, im Lichte neuester Entwicklungen betrachtet, um eine zentrale Problemstellung der Wissenschaftsphilosophie überhaupt handelt. Es geht um die Rolle eines apriorischen Elementes in der wissenschaftlichen Erkenntnis.

4. Ein unausgetragener Disput. Der Begriff des (synthetischen) A priori ist ein Erbe der kantischen Wissenschaftsphilosophie. In der Wissenschaftsphilosophie des 20. Jahrhunderts erlebte es eine sehr wechselhafte Einschätzung. Während manche "wissenschaftlichen" 
Philosophen wie Carnap und Reichenbach zu Beginn ihrer philosophischen Karriere noch eine Art konstitutives, aber nicht mehr apodiktisches a priori vertraten, lehnten andere, wie etwa Schlick, dieses Erbstück der Wissenschaftstheorie des 19. Jahrhundert rundweg ab.

Mit der Konstitution des Wiener Kreises und der Berliner Gruppe Ende der zwanziger Jahre war das Thema scheimbar im negativen Sinne abgeschlossen: ein wie immer geartetes a priori, das mehr als Konvention zu sein beanspruchte, lehnten die Mitglieder beider Gruppen einhellig ab. Um so überraschender mag es für die Organisatoren des Pariser Kongresses gewesen sein, daß die Thematik des Apriori bei den Diskussionen in Paris noch einmal auftauchte. So berichtet Neurath in seinem Kongreßbericht, daß der italienische Wissenschaftsphilosoph Enriques "die bloße Ablehnung des kantischen apriori nicht ausreichend [gefunden habe], sondern an dessen Stelle gewisse, innerhalb eines bestimmten Rahmens Grundsätze des Denkens setzen [wollte]" (Neurath 1935, 387). Dagegen hätten Frank, Reichenbach und andere wesentliche Bedenken geäußert. Enriques' Kritiker wendeten ein, aus einem variablen apriori ließen sich überhaupt keine sinnvollen Konsequenzen mehr ziehen, und was Enriques Forderungen der Vernunft nenne, könne man als Konvention, Gewohnheit, oder allgemeines aposteriori auffassen. Und außerdem, so wendeten die Anhänger des Wiener Kreises ein, daß die Vernunft ihre aprioris ändere, gehöre ins Gebiet der Geschichte der Wissenschaften, und nicht ins Gebiet der Wissenschaftslogik (ibidem). Vertreter abweichender Auffassungen (außer Enriques) erwähnt Neurath in seinem Bericht nicht. Das ist insofern merkwürdig, als Morris im selben Band der Erkenntnis, in dem Neuraths Kongreßbericht veröffentlicht wurde, seine Arbeit The Relation of the Formal and the Empirical Sciences within Scientific Empiricism (Morris 1935, LPS, IV) veröffentlichte, in der er explizit für die Existenz eines "variablen A priori" in der wissenschaftliches Erkenntis plädierte:

There is at any moment, for thinking beings, an a priori in the sense of a set of meanings in terms of which empirical data are approached, and logical analysis may be regarded as following the structural lines of the a priori which support inference. This a priori, however, undergoes change through contact with the new data which are encountered through its use, and through changes in human interests and purposes. With every such change the a priori is altered, and new content for logical analyis is provided. Acting on the new set of meanings brings 
new data and new purposes, which in turn affect the content and structure of the a priori. And so the spiral process continues. (Morris 1935, 10, und LPS, 51 )

Morris stand im Pragmatismus mit dieser Konzeption eines variablen analytischen apriorischen Elementes im wissenschaftlichen Wissens im Pragmatismus keineswegs allein. Bereits in den frühen zwanziger Jahren hatte C.I. Lewis einen ähnlichen Ansatz entwickelt und in seinem Hauptwerk Mind and the World Order (Lewis 1929) zu einer umfassenden Theorie ausgearbeitet. Man kann also mit einigem Recht behaupten, daß das Problem eines apriorischen Elementes in der wissenschaftlichen Erkenntnis einen wesentlichen Unterschied zwischen logischem Empirismus und Pragmatismus markierte.

Die Thematik des variablen A priori wird noch interessanter durch die folgende Tatsache. Vor einiger Zeit hat Michael Friedman darauf hingewiesen, daß Carnap in Logische Syntax der Sprache (Carnap 1934) Reichenbachs ursprüngliche Idee eines konstitutiven relativierten a priori in neuer Form, nämlich als L-Regeln oder analytische Sätze, wieder aufgenommen habe (cf. Friedman 2000, 69). Carnap scheint sich dieser Tatsache damals jedoch nicht bewußt gewesen zu sein, jedenfalls machte er offenbar keine Anstalten, die hier vorhandene Affinität zu Morris' (oder Lewis') Ansatz herauszustellen (zumindest berichtet Neurath nichts darüber). Mit dieser Haltung stand er nicht allein: auch Neurath, ein strikter Gegner jedweder kantianischer Reminiszenzen in der Wissenschaftstheorie, hatte seinerzeit Carnaps Logische Syntax enthusiastisch, wenn auch wohl nicht sehr sachkundig begrüßt und dieses Werk keineswegs als Wiederaufnahme kantianischer Philosopheme kritisiert (cf. Uebel 2008, 68f.). Die von Friedman herausgearbeiteten kantianischen Aspekte der Logischen Syntax der Sprache sind also offenbar beim Erscheinen dieses Werkes weder vom Autor selbst noch von seinen Zeitgenossen als solche erkannt worden. Damit soll keineswegs behauptet werden, es gäbe sie nicht. Es ist ja durchaus möglich, daß wir heute die Motive und philosophiehistorischen Dependenzen früherer Denker besser verstehen als sie selbst zu ihrer Zeit. Darüber hinaus ist denkbar, daß den Mitgliedern des Wiener Kreises aus wissenschaftspolitischen Gründen wenig daran gelegen war, kantianische Anklänge ihrer Gedankengänge an die große Glocke zu hängen. Jedenfalls blieb das Themenfeld der Rolle des a priori in der empirischen Erkenntnis zur Mitte des 20. Jahrhunderts von logisch-empiristischer Seite unbestellt und ziemlich ausschließlich der pragmatistischen Wissenschaftstheorie überlassen. 
5. Zur Entwicklung von Morris' Projekt nach 1935. Der Pariser Kongreß war nur der erste in einer Reihe "internationaler Kongresse für Einheit der Wissenschaft". ${ }^{3}$ Morris' Versuche ein Programm für eine attraktive Synthese von Pragmatismus und logischem Empirismus zu formulieren, endeten natürlich nicht in Paris, oder besser gesagt mit der Veröffentlichung von LPS zwei Jahre später. Im Gegenteil, man kann sagen, daß in den Jahrzehnten nach dem Pariser Kongreß dieses Projekt einer Synthese einen Gutteil seiner philosophischen Lebensarbeit ausmachte, obwohl es sowohl bei den Pragmatisten wie bei den logischen Empiristen auf immer weniger Interesse stieß. Erwähnenswert in diesem Zusammenhang sind insbesondere der Vortrag Semiotic, The Socio-Humanistic Sciences, and the Unity of Science, den Morris 1939 auf dem vorletzten (fünften) Kongreß für Einheit der Wissenschaft in Harvard hielt, und sein Beitrag Logical Empiricism and Pragmatism (Morris 1963) zum Carnap gewidmeten Schilpp-Band der Reihe Living Philosophers.

Von Morris' Vortrag Semiotic, The Socio-Humanistic Sciences, and the Unity of Science (SHC) existiert ein nahezu druckfertiges Manuskript, das im Band 9 der Zeitschrift Erkenntnis erscheinen sollte, wozu es aber aufgrund des Krieges nicht mehr kam. ${ }^{4}$ Die Intention und den wesentlichen Inhalt seines für den fünften Kongreß geplanten Beitrages hatte Morris schon ein Jahr zuvor in dem kurzen Artikel The Unity of Science Movement and the United States (Morris 1938) angekündigt. Was er plante, war nicht mehr und nicht weniger als Neuraths Programm der Einheitswissenschaft eine genuin "amerikanische" Wendung zu geben, was dazu beitragen sollte, seine Einseitigkeiten und Unzulänglichkeiten zu überwinden. Die Tatsache, daß der Kongreß auf amerikanischen Boden in Harvard stattfinden würde, schien inm dafür eine besonders günstige Gelegenheit zu bieten:

The United States seems, from a sociological point of view, to be singularly prepared to play an important place in the incorporation of the socio-humanistic studies into the unity of science movement. (Morris 1938, 27)

Dafür, daß Amerika hier eine besondere Rolle spielen konnte, führte er mehrere Gründe an. Zum einen seien die USA in der Psychologie und der Soziologie führend, überdies seien die USA das Land, in dem eine empirische Werttheorie entwickelt worden sei, und schließlich bilde der Pragmatismus ein besonders geeignetes philosophisches Umfeld, diese neue Art einer

\footnotetext{
${ }^{3}$ Ausführliche Informationen über Umstände, Themen und Teilnehmer aller Kongresse finden sich in Stadler (1997).
} 
umfassenden Einheitswissenschaft auf den Weg zu bringen. Ob diese amerikanische Variante von Einheitswissenschaft, die natürlich viele Aspekte von Morris' Pariser Programm wieder aufnahm, die uneingeschränkte Zustimmung Neuraths gefunden hat, kann man bezweifeln.

Das Programm des fünften Kongresses spiegelt diese von Morris intendierte Verschiebung des Schwerpunktes in Richtung auf eine stärkere Betonung der Geisteswissenschaften in einigen Beiträgen durchaus wieder, so z.B. in Gomperz' Vortrag Unified Science and Value (Gomperz 1975), der nach dem Krieg in der "neuen Erkenntnis” veröffentlicht wurde.

Auch wenn man also den Harvard-Kongreß als persönlichen Erfolg von Morris ansehen kann, hat er insgesamt den Niedergang der "Unity of Science"-Bewegung in den USA nach dem Ende des Krieges und nach Neuraths Tod 1945 nicht aufhalten können (cf. Reisch 2005). Das Interesse an Morris' Programm schwand, wozu wohl auch die Tatsache beitrug, daß Morris' philosophischer Stern nach 1945 im Sinken begriffen war, da weder die "richtigen" Pragmatisten wie Dewey noch die Logischen Empiristen ihn und seine semiotischen Aktivitäten noch allzu ernst nahmen (cf. Reisch 2005).

Einen letzten Versuch, die Beziehungen zwischen amerikanischem Pragmatismus und logischen Empirismus à la Carnap zu verbessern und eventuell nur verbale Differenzen und Mißverständnisse auszuräumen, unternahm Morris in dem Artikel Logical Empiricism and Pragmatism (Morris 1963), der als Beitrag zu dem Carnap gewidmeten Schilppband The Philosophy of Rudolf Carnap erschien. Wie schon früher betrachtete er als neuralgischen Punkt der Auseinandersetzung das Thema "Werte":

It has been a central tenet of the pragmatists, no matter how great their other differences, that judgments of values are empirical in nature, and so have a cognitive or theoretical character amenable in principle to control by scientific method. ... [T] he pragmatists have believed that judgments of value as well as the statements of science conform to the pragmatic maxim and are meaningful in the same sense. Carnap has certainly affirmed the opposite. (Morris 1963, 94)

Trotz dieses "offiziellen" schroffen Gegensatzes glaubte er eine Möglichkeit gefunden zu haben, Carnap zumindest indirekt oder "inoffiziell” für einen kognitivistischen Standpunkt in der Ethik "retten" zu können. Er wies nämlich darauf hin, daß Carnap zumindest im Aufbau eine Position vertreten hatte, wonach “Werte” analog empirischen Qualitäten durch geeignete

\footnotetext{
${ }^{4}$ Ich danke Friedrich Stadler, daß er mir eine Kopie des Textes zur Verfügung gestellt hat.
} 
Werturteile konstituiert würden. Diese Methode ähnele dem Verfahren von Lewis, der in An Analysis of Knowledge and Valuation (Lewis 1946) Werturteile als empirischen Urteilen analoge Urteile über Werterfahrungen konzipiert habe. Darüber hinaus reduziere auch Carnap die Philosophie nicht auf Wissenschaftslogik, da er die Aufgabe der Philosophen darin erblicke, “Vorschläge zu machen”. Das aber sei, wie die Pragmatisten immer wieder betont hätten, eine wertgeleitete Tätigkeit (vgl. Morris 1963, 97). In seiner Replik zeigte sich Carnap von diesen Argumenten wenig beeindruckt. Morris' Verweis auf die Werttheorie im Aufbau ignorierte er völlig. Ansonsten machte er Morris nur die ziemlich nichtssagende Konzession: “I am inclined to agree with Morris that the difference between by view and that of the pragmatists is not as large as it might appear at first glance" (Carnap 1963, 862).

Insgesamt also ein eher unbefriedigendes Fazit eines Wettbewerbs zwischen logischempiristischem Pragmatismus, Wissenschaftslogik und Neuraths Einheitswissenschaft, der 1935 seinen Ausgang in Paris genommen hatte.

6. Ein Pyrrhussieg. Morris' Projekt eines umfassenden logisch-empiristischen Pragmatismus als einer Synthese von amerikanischem Pragmatismus und logischem Empirismus ist Programm geblieben. Im Rahmen der analytischen Wissenschaftsphilosophie setzte sich um die Mitte des 20. Jahrhunderts weitgehend Carnaps Wissenschaftslogik als leitendes Paradigma durch, das Enzyklopädieprojekt hingegen fiel nach dem Tod seines Protagonisten Neurath für lange Zeit der Bedeutungslosigkeit anheim und findet erst neuerdings wieder mehr Beachtung, ohne daß aber eine Wiederaufnahme dieses Ansatzes in der aktuellen wissenschaftsphilosophischen Situation der zur Debatte stünde.

Aus dem Wettbewerb der drei in Paris vorgetragenen wissenschaftsphilosophischen Programme der Wissenschaftslogik, des Enzyklopädismus, und des wissenschaftlichen Pragmatismus ist also ohne Zweifel Carnaps Wissenschaftslogik als Sieger hervorgegangen. Dieser Sieg erwies sich jedoch bald als Pyrrhussieg, der der Wissenschaftsphilosophie und der wissenschaftlichen Philosophie insgesamt erheblich schadete. Er trug dazu bei, daß für längere Zeit politische, gesellschaftliche und allgemein praktisch-relevante Aspekte des wissenschaftlichen Wissens aus dem Horizont der mainstream Wissenschaftsphilosophie verschwanden. Ein Beleg dafür ist die Tatsache, daß seit den sechziger Jahren diese "klassische" logisch-orientierte Wissenschaftsphilosophie gegenüber historistisch orientierten 
Ansätzen in die Defensive geriet, wozu ironischer Weise das noch in der International Enzyclopedia of Unified Science erschienene Werk Kuhns einen wesentlichen Beitrag leistete. Auch Morris' Bemühungen, den Pragmatismus dem logischen Empirismus anzunähern, fanden von seiten der Pragmatisten immer weniger Interesse (cf. Reisch 2005), stattdessen entledigten sich neopragmatistische Strömungen wie der Ansatz Rortys vollständig der ursprünglichen Wissenschaftsorientierung des klassischen Pragmatismus von Peirce, Lewis, und Morris (cf. Rorty 1982, 2007). Insgesamt also eine eher düstere Bilanz.

Vor kurzem hat nun Thomas Uebel die These aufgestellt, die zunehmende Isolierung der traditionellen logisch-empiristischen Wissenschaftsphilosophie seit 1960 beruhe eigentlich auf einem Mißververständnis: es sei schlicht falsch, logischen Empiristen wie Carnap zu unterstellen, sie hätten einer auf die Wissenschaftslogik fixierten Wissenschaftsphilosophie das Wort geredet. In Wirklichkeit habe der logische Empirismus eine viel offenere Konzeption vertreten, der zufolge die logisch-empiristische Wissenschaftsphilosophie eben nicht nur Wissenschaftslogik im engen Sinne umfaßte, sondern auch empirische Disziplinen wie Wissenschaftsgeschichte, Wissenschaftssoziologie, Wissenschaftspragmatik und möglicherweise noch anderes einschloß (Uebel 2013, 253). Carnaps wissenschaftsphilosophischer Ansatz sei durchaus kompatibel mit Neuraths "Gelehrtenbehavioristik" und Franks "Wissenschaftspragmatik" gewesen (ibid.). Kurzum, der "linke Flügel des Wiener Kreises", betrachtet als ein Kollektivsubjekt, habe für eine "bipartite metatheory" der Wissenschaft (BPM) als Nachfolgedisziplin der traditionellen Wissenschaftschaftsphilosophie plädiert, die keineswegs, wie oft behauptet, die pragmatischen und praktischen Aspekte der Wissenschaft vernachlässigt habe. BPM habe nur, und das völlig legitim, klar zwischen logischen Aspekten der Wissenschaft auf der einen, und historischen, soziologischen und anderen empirisch zu untersuchenden Aspekten der Wissenschaft unterschieden. Wenn ein Teil des Kollektivsubjektes "linker Flügel des Wiener Kreises", etwa Carnap, sich vorwiegend mit Wissenschaftslogik befaßt habe, sei dies schlicht das Ergebnis einer zweckmäßigen, im Prinzip aber bedeutungslosen Arbeitsteilung gewesen.

Aus dieser Perspektive lasse sich, so Uebel, eine enge Beziehung zwischen BPM und Morris' Programm feststellen:

while Morris's program for the unification of pragmatism and logical empiricism was by no means adopted in toto [by Carnap, Neurath, and Frank, T.M.], significant aspects of it were - namely, precisely those (and only those) aspects 
that sustain the blueprint for the bipartite conception of philosophy as metatheory. (Uebel 2013, 524)

Uebel zufolge waren also die logischen Empiristen gegenüber Morris' pragmatistischen Avancen gar nicht so unzugänglich wie ich es hier dargestellt habe. BPM sei vielmehr so etwas wie der logisch-empiristisch akzeptable Kern von Morris' ansonsten stark metaphysisch kontaminiertem Programm eines "wissenschaftlichen Empirizismus" gewesen. Uebel geht sogar so weit, BPM als ein Projekt zu charakterisieren, das weiter zu erforschen auch für die zeitgenössische Wissenschaftsphilosophie lohnend sein könnte:

... [I]t strikes me as not impossible that this conception of a bipartite metatheory should prove useful in the further development of what nowadays would be called "deflationary" philosophy of science. So here's one "path not taken" that might, for collegues with deflationist sympathies, merit further considerations. (Uebel 2010, 25).

Aus verschiedenen Gründen, die hier nicht erörtert werden können, teile ich Uebels Wertschätzung für BPM nicht - zum einen scheint mir BPM eher der Schatten einer Theorie zu sein denn eine gehaltvolle philosophische Theorie, zum anderen verweigert sich BPM hartnäckig der bereits erwähnten Lewis'schen pragmatistischen Grundthese, der zufolge "knowledge, action, and evaluation are essentially connected", und stellt stattdessen "Theorie" und "Praxis" unverbunden neben einander. Wie dem auch sei, wer BPM als einen ausssichtsreichen Ansatz ansieht, dessen Erkundung auch für die zeitgenössische Wissenschaftsphilosophie lohnen könnte, dürfte eigentlich auch Morris' weitaus anspruchsvolleres Programm einer Synthese von logischem Empirismus und Pragmatismus nicht unbeachtet links liegen lassen. Ohne also einer schlichten Wiederaufnahme von Morris' Pariser Programm das Wort zu reden, kann man wohl behaupten, daß Morris' Programm auf einige Desiderata einer umfassenden, politisch und gesellschaftlich verantwortungsvollen Wissenschaftsphilosophie hinweist, die auch für die zeitgenössische wissenschaftsphilosophische Diskussion nichts von ihrer Aktualität eingebüßt haben.

Literatur: 
Apel, K.O., 1974, Zur Idee einer transzendentalen Sprachpragmatik. Die Dreistelligkeit der Zeichenrelation und die "abstractive fallacy" in den Grundlagen der klassischen Transzendentalphilosophie und der sprachanalytischen Wissenschaftslogik, in J. Simon (Hrg.), Aspekte und Probleme der Sprachphilosophie, Freiburg/Breisgau, Alber, 283 - 326.

Carmap, R., 1934, Die Aufgabe der Wissenschaftslogik, Einheitswissenschaft Bd. 3 . Gerold, Wien. Französische Übersetzung Le problème de la Logique de Science. Actualités Scientifiques, vol. 291, Hermann, Paris 1935.

Carnap, R., 1935, Philosophy and Logical Syntax, London, Kegan, Trench and Trubner.

Carnap, R., 1936, Von der Erkenntnistheorie zur Wissenschaftslogik, Actes du Congrès International de Philosophie Scientifique I, Philosophie Scientifique et Empirisme Logique, Actualités Scientifiques et Industrielles 388, Hermann, Paris, 36 - 41.

Carnap, R., 1963, Charles Morris on Pragmatism and Logical Empiricism, in P. A. Schilpp, The Philosophy of Rudolf Carnap (ed.), Chicago and LaSalle, Open Court, 860 - 862.

Friedman, M., 1999, Reconsidering Logical Positivism, Cambridge, Cambridge University Press. Friedman, M., 2003, Kuhn and Logical Empiricism, in T. Nickles (ed.), Thomas Kuhn, Cambridge, Cambridge University Press, 19 - 44.

Gomperz, H., 1975 (1939), Unified Science and Value, Erkenntnis 9, 5 - 10.

Lewis, C.I., 1946, An Analysis of Knowledge and Valuation, Chicago and LaSalle, Open Court. Mormann, T., 2007, Carnap's Logical Empiricism, Values, and American Pragmatism, Journal of General Philosophy of Science, 38/1, 127-146.

Mormann, T., 2010, History of Philosophy of Science by other Means? Comment on Thomas Uebel, in Friedrich Stadler, 2010 (ed.) together with Dennis Dieks, Wenceslao J. Gonzáley, Stephan Hartmann, Thomas Uebel, Marcel Weber, The Philosophy of Science in a European Perspective, Springer, 29 - 39.

Morris, C.W., 1935, The Relation of the Formal and Empirical Sciences within Scientific Empiricism, Erkenntnis 5, 6 -16. (LPS, Chapter IV, 46 - 55.

Morris, C.W., 1936, Semiotic and Scientific Empiricism, Actes du Congrès International de Philosophie Scientifique I, Philosophie Scientifique et Empirisme Logique, Actualités Scientifiques et Industrielles 388, Hermann, Paris, $42-56$.

Morris, C.W., 1937, Logical Positivism, Pragmatism and Scientific Empiricism, Exposés de Philosophie Scientifique 449, Hermann, Paris. (LPS)

Morris, C.W., 1938, The Unity of Science Movement and The United States, Unity of Science Forum (Synthese), November 1938, 25 - 29. 
Morris, C.W., 1939, Semiotic, The Socio-humanistic Sciences, and the Unity of Science, unpublished ms., Paper for the 5th International Congress for the Unity of Science, Cambridge/Massachusetts, September 1939.

Morris, C.W., 1960, On the History of The International Encyclopedia of Unified Science, Synthese 12, $517-521$.

Morris, C.W., 1963, Pragmatism and Logical Empiricism, in P.A. Schilpp (ed.) The Philosophy of Rudolf Carnap, Chicago and LaSalle, Open Court, 87 - 98.

Nemeth, E., Roudet, N., 2005, Paris - Wien, Enzyklopädien im Vergleich (Hrg.), Wien und New York, Springer.

Neurath, O., 1935, Erster Internationaler Kongress für Einheit der Wissenschaft in Paris 1935, Erkenntnis 5, 377 - 406.

Neurath, O., 1936, Einzelwissenschaften, Einheitswissenschaft, Pseudorationalismus, Actes du Congrès International de Philosophie Scientifique I, Philosophie Scientifique et Empirisme Logique, Actualités Scientifiques et Industrielles 388, Hermann, Paris, 36 - 41.

Reisch, G. A., 2005, How the Cold War Transformed Philosophy of Science. To the Icy Slopes of Logic, Cambridge, Cambridge University Press.

Rorty, R., 1982, Consequences of Pragmatism, Minneapolis, University of Minnesota Press.

Rorty, R., 2007, Philosophy as Cultural Politics, Philosophical Papers volume 4, Cambridge, Cambridge University Press.

Soulez, A., 2005, Der Neurath-Stil, oder: der Wiener Kreis, Rezeption und Rezeptionsprobleme auf den Kongressen 1935 und 1937 in Paris, in E. Nemeth und N. Roudet (Hrg.), 121150.

Stadler, F., 1997, Studien zum Wiener Kreis, Frankfurt/Main, Suhrkamp Verlag.

Uebel, T., 2010, Some Remarks on Current History of Analytic Philosophy of Science, in Friedrich Stadler, 2010 (ed.) together with Dennis Dieks, Wenceslao J. González, Stephan Hartmann, Thomas Uebel, Marcel Weber, The Philosophy of Science in a European Perspective, Springer, $13-28$.

Uebel, T., 2012, The Bipartite Conception of Metatheory and the Dialectical Conception of Explication, in P. Wagner (ed.), Carnap's Ideal of Explication and Naturalism, 117 - 130.

Uebel, T., 2013, Pragmatics in Carnap and Morris and the Bipartite Metatheory Conception, Erkenntnis 78(3), $523-546$. 